\title{
High-speed imaging the effect of snap-off distance and squeegee speed on the ink transfer mechanism of screen-printed carbon pastes
}

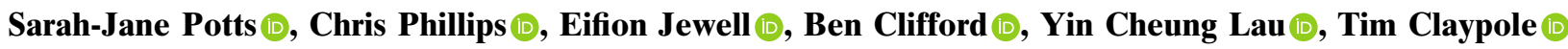

(C) The Author(s) 2019

\begin{abstract}
Screen printing is the most widely used process in the production of printed electronics due to its ability to consistently transfer inks containing a wide range of functional materials onto a range of substrates. However, despite its extensive use, the mechanism by which the ink is transferred through the mesh and onto the substrate is not fully understood. Existing theories are contradictory and lack experimental validation. Therefore, high-speed imaging was used in combination with a screen-printing simulation rig that was designed to provide good optical access to study ink deposition during the screen-printing process. The variation in the four stages of ink flow through the screen, described in the theory by Messerschmitt, has been quantified with respect to changes in snap-off
\end{abstract}

Electronic supplementary material The online version of this article (https://doi.org/10.1007/s11998-019-00291-6) contains supplementary material, which is available to authorized users.

S.-J. Potts ( $\varangle)$, C. Phillips, B. Clifford,

Y. C. Lau, T. Claypole

Welsh Centre for Printing and Coating, College of Engineering, Swansea University, Bay Campus, Crymlyn

Burrows, Swansea SA1 8EN, UK

e-mail: Sarah-Jane.Potts@swansea.ac.uk

C. Phillips

e-mail: C.O.Phillips@swansea.ac.uk

B. Clifford

e-mail: B.R.Clifford@Swansea.ac.uk

Y. C. Lau

e-mail: Y.C.Lau@swansea.ac.uk

T. Claypole

e-mail: T.C.Claypole@swansea.ac.uk

E. Jewell

SPECIFIC, College of Engineering, Swansea University, Bay Campus, Crymlyn Burrows, Swansea SA1 8EN, UK e-mail: E.Jewell@Swansea.ac.uk distance and squeegee speed. Analyses of the images were compared with measurements of the ink properties and corroborated with analyses of the prints. This has provided a better understanding of the mechanism by which the ink transfers from the mesh to the substrate and subsequently separates in screen printing. This could be used as the basis for the development of predictive algorithms, as well as to improve the understanding of how to optimize print quality and performance.

Keywords Ink transfer, Rheology, Printed electronics, Carbon inks, High-speed imaging

\section{Introduction}

Screen-printed carbon inks and pastes are widely used in the manufacturing of a range of printed electronics applications due to their electrical conductivity and relatively low cost. These include resistive heaters, ${ }^{1}$ electrochemical sensors, ${ }^{2}$ printed batteries,${ }^{3}$ perovskite photovoltaics, ${ }^{4,5}$ energy harvesting in the form of printed pyroelectrics, ${ }^{6}$ and thermoelectrics. ${ }^{7}$ Deposition quality is an essential component in the performance of these products. Research has been conducted to identify the effects of various press parameter settings as well as ink formulation on screen-printed carbon inks and pastes to optimize print quality and electrical performance. ${ }^{1,8,9}$ The effect of mesh material and geometry was found to be relatively consistent for a range of inks, with finer meshes leading to reduced film thickness but improved definition, which is preferable for fine feature printing. ${ }^{9-11}$ Parameters such as squeegee hardness, angle, and geometry have also been found to have consistent effects on a range of inks, where softer squeegees at shallow angles were found to produce thicker deposits. ${ }^{12}$ However, squeegee pressure, snap-off distance (the distance between the 
screen and the substrate) and print speed have not demonstrated consistent trends and have been shown to vary with the rheology of the ink. ${ }^{12-18}$ Yet, there has only been limited fundamental research to establish the underpinning science of screen printing. The fundamental mechanisms behind this process remain poorly understood.

It has been a challenge to develop predictive models of the screen-printing process due to limitations in modeling the rheological properties of screen-printing inks. ${ }^{19-2}$ This limits the understanding of the physical mechanisms of ink transfer which occur during screen printing. Computational fluid dynamics (CFD) models have been developed to assess both the Newtonian and non-Newtonian inks $\mathrm{s}^{20,22,23}$ based on mechanisms proposed by Riemer ${ }^{19-21,24,25}$ and Messerschmitt ${ }^{26}$ to predict how the ink flows and separates during screen printing. Riemer ${ }^{24}$ likened the openings in the mesh to capillaries, forcing the ink into the mesh in accordance with the Hagen-Poiseuille law. ${ }^{20,24}$ This provided a way of calculating the volumetric flow rate through the holes in the mesh. Riemer ${ }^{20}$ also suggested that there were two key forces occurring during separation, where the strands in the mesh are pulled up by the tension acting on the mesh but countered by a downward force acting between the ink and mesh. As the surface area of the mesh containing the ink is greater than that of the substrate being printed onto, Messerschmitt ${ }^{26}$ argued that the adhesive forces between the substrate and the ink could not be sufficient to transfer all ink to the substrate. Instead, Messerschmitt suggested that although the separation forces would be insufficient to break the adhesion between the ink and the mesh, they could induce a flow which would create a shearing action within the ink resulting in cohesive failure. ${ }^{22}$ This process was summarized as four key stages of ink transfer, namely adhesion, extension, flow and finally separation where the ink split with some remaining on the surface of the mesh and the remainder on the surface of the substrate, in the form of the print. ${ }^{26}$ Based on the work by Riemer ${ }^{20}$ and Messerschmitt, ${ }^{26}$ Kapur et al. ${ }^{27}$ produced a CFD model, based on the Landau-Levich equation and capillary number, to calculate the volume of ink transferred during the screen-printing process. The model consisted of two main flow regimes, including shear flow occurring over the mesh strand, with extensional flow occurring between the mesh strand and substrate, and a filament breakup mechanism based on Messerschmitt's four stages of ink transfer. A limitation in this model is that it is based on contact screen printing, where the screen is lifted from the substrate after the full print stroke has been conducted, and there would only be a vertical strain and strain rate affecting the ink separation. However, in practice screen printing is typically conducted as an off-contact process, with a snap-off distance between the mesh and substrate, which would also induce horizontal forces due to the angular contact of the mesh and substrate which would move at a rate determined by the squeegee speed.

$\mathrm{Xu}$ and Willenbacher ${ }^{23}$ conducted a high-speed video imaging study on fine line screen-printed $\mathrm{ZnO}$ pastes. The ink deposition was imaged with a camera fixed directly below a glass substrate, looking up at the print. Three main regions of ink transfer were identified, including a pre-injection zone, where the ink deposited ahead of the squeegee, the cling zone from the nip contact point to snap-off position as defined by Riemer, ${ }^{20}$ and finally the paste spreading zone, which is where the ink slumps after it has been deposited, which if too long can cause loss of resolution. Although these findings provided an insight into the size of the cling zone and quantity of paste spreading after print deposition, they did not confirm whether the separation models suggested by Riemer and Messerschmitt were appropriate. Overall, there is a lack of knowledge of the process mechanism and whether it is influenced by press parameter settings. Print defects such as mesh marking, which causes regular features corresponding with the frequency of the mesh, as well as pin holes can have serious repercussions for printed electronics.

The investigation had three primary objectives. Firstly, it aimed to develop qualitative methods to enable the identification and measurement of key fluid mechanisms at the screen-substrate interface. Secondly, it aimed to investigate the impact of important process parameters on the print deposit and explain their influence through understanding the screen to substrate fluid mechanisms. Thirdly, it aimed to investigate whether laboratory ink characterization could be used to explain and subsequently predict the changes in ink deposit.

High-speed imaging was conducted on a screenprinting visualization rig, to analyze how the transfer mechanism from the mesh to the substrate of a commercial carbon ink altered with snap-off distance and squeegee speed. The effect of these two parameters is poorly understood. Topographic analyses of the resulting prints were used to assess whether changes in the ink deposition mechanism affected the resulting ink transfer and print quality. Shear rheometric analyses were used to establish the viscoelastic properties of the ink. The Capillary Breakup Extensional Rheology (CaBER) technique was used to evaluate the relative amounts of material directed to bottom or top interfaces and flow characteristics as the ink is pulled apart. This is analogous to the extent to which the ink is either transferred to a substrate or retained by the screen mesh. Changes in the mode of capillary thinning and breakup with alterations in strain and strain rate were related to those seen in filamentation occurring during the printing process. However, in screen printing there is the additional complexity of the effects of the mesh strands and the shearing action occurring in both the print direction and the vertical separation occurring perpendicular to the print direction due to the angle between the mesh and substrate, making it 
hard to evaluate the relative effects of vertical or horizontal forces and the separation mechanisms.

\section{Materials and methods}

\section{Rheological and extensional testing}

The ink was a commercial carbon ink from Gwent Electronics Materials [GEM C2150317D3 carbon paste (contains a blend of graphite and carbon black, with a solid content of $38-42 \%$ )]. Rheological evaluation was carried out using a combination of shear, viscoelastic and extensional measurements. Shear viscosity measurements were carried out on a Malvern Bohlin rotational rheometer (Gemini Bohlin Nano, Malvern Instruments) with a $2^{\circ} 20-\mathrm{mm}$ stainless steel cone and a plate held at $25^{\circ} \mathrm{C}$. Ink viscosity was measured as the shear rate was gradually increased to $100 \mathrm{~s}^{-1}$ and then reduced back to $1 \mathrm{~s}^{-1}$. Viscoelastic measurements were carried out on a Malvern Kinexus Pro rheometer (Malvern Instruments) with a $40-\mathrm{mm}$ roughened plate and roughened parallel plate (to minimize the effect of slip). Amplitude (strain) sweep measurements were conducted to establish the linear viscoelastic range at $0.1,1$ and $10 \mathrm{~Hz}$. Then using a stress within the established linear viscoelastic region, a frequency sweep from 0.1 to $10 \mathrm{~Hz}$ with 30 logarithmically spaced measurements was conducted.

Extensional testing of the ink was assessed using capillary breakup extensional rheology (CaBER) testing methods, where ink is rapidly separated to form a liquid bridge between the two plates. The subsequent evolution of the liquid bridge after it has been separated to a set distance until subsequent breakage under the action of capillary pressure was monitored and compared. ${ }^{28,29}$ Ink samples were placed between two parallel 3-mm-diameter stainless steel plates held at a 1-mm gap, where the upper plate was then moved upwards at a constant velocity. The following change in minimum diameter over time until separation point was then recorded and measured. To assess the effect of separation distance and speed on the ink separation, this gap was extended by 3,5 and $7 \mathrm{~mm}$ by moving the upper plate vertically upwards. For each gap, this was performed at constant velocities of 20,60 and $100 \mathrm{~mm}$ $\mathrm{s}^{-1}$. The separation distances used for the $3-\mathrm{mm}$ cylinders were larger than the snap-off distances used in the screen-printing rig, as the 3-mm cylinders would produce far larger filaments than the $200-\mu \mathrm{m}$ lines being printed on the rig. The extension and separation of the cylindrical liquid bridges were captured using a high-speed camera (Photron FastCam Mini HighSpeed Camera) at a frame rate of 125 frames per second. The images were used to assess the change in minimum radius over time and the relative material flow from bottom to top plates in terms of crosssectional area of ink split between the plates, as well as length to separation point from the bottom plate.

\section{Screen-Printing visualization method}

Printing was conducted on a screen-printing visualization rig, which allowed the screen-substrate separation to be captured during the print via the high-speed camera (Fig. 1). The rig used a small screen [100 $\mathrm{mm} \times 130 \mathrm{~mm}$ polyester mesh at $22.5^{\circ}$ with 61 threads per $\mathrm{cm}, 64 \mu \mathrm{m}$ thread diameter and $12 \mu \mathrm{m}$ emulsion over mesh (EOM)] (supplied by MCI Precision Screens Ltd.), which enabled imaging at the camera's focal length $(80 \mathrm{~mm})$. Squeegee motion in the print direction ( $x$-axis) and vertical movement perpendicular to the print direction ( $z$-axis) were controlled by stepper motors powering lead screws on linear actuators. It was programmed using Arduino with Grbl Controller 3.0 (open source) software to set the speed and distance of the movement. This allowed the squeegee to be brought into contact with the screen and then brought across the screen to transfer the ink. A 65-70 shore A hardness diamond squeegee was used. The squeegee had a $10 \mathrm{~mm} \times 10 \mathrm{~mm}$ profile and was $10 \mathrm{~mm}$ in length to minimize deflection on the screen. In the experiment, the snap-off distance (distance between screen and substrate) and squeegee speeds were both varied over three settings, with snapoff distances of $1.125,1.475$ and $1.825 \mathrm{~mm}$ and squeegee speeds of $100 \mathrm{~mm} \mathrm{~min}^{-1}\left(1.7 \mathrm{~mm} \mathrm{~s}^{-1}\right), 300$ $\mathrm{mm} \min ^{-1}\left(5.0 \mathrm{~mm} \mathrm{~s}^{-1}\right)$ and $500 \mathrm{~mm} \mathrm{m^{-1 }}(8.3 \mathrm{~mm}$ $\mathrm{s}^{-1}$ ). The substrate was PET [polyethylene terephthalate-Melinex ${ }^{\circledR}$ 339, DuPont Teijin Films (175 $\mu \mathrm{m}$ thickness) opaque white]. The samples were dried at room temperature. The print image consisted of a continuous $200-\mu \mathrm{m}$-wide line in the direction of squeegee travel. This width was selected as it produced clearer and more consistent prints than those conducted through the narrower lines. Having only one gap between threads across the width of the line, there were only single filamentations separating across the width of the line.

Imaging of the print cycle was conducted of the interface between the screen and the substrate. It was conducted with the same camera as used in extensional testing, at 125 frames per second with $5 \times$ magnification. A 10,000 lux lamp was placed directly behind the screen-printing rig to provide sufficient backlighting for high contrast imaging. Camera images were assessed using Image ${ }^{30}$ to measure the lengths of different regions of the print cycles, based on the four regions of flow identified by Messerschmitt. ${ }^{26}$ These four regions were split up into two quantifiable lengths. Additionally, the length of the paste flow ahead of the squeegee was assessed to make up the full contact region, which consisted of the total duration where the ink is simultaneously in contact with the mesh and substrate. The lengths of these flow regions were then measured every $0.024 \mathrm{~s}$ (around every third frame) across the print run, where the full contact region (where the ink was in simultaneous contact with the mesh and substrate) could be seen. This produced around 15 measurement sets for each print run, from 

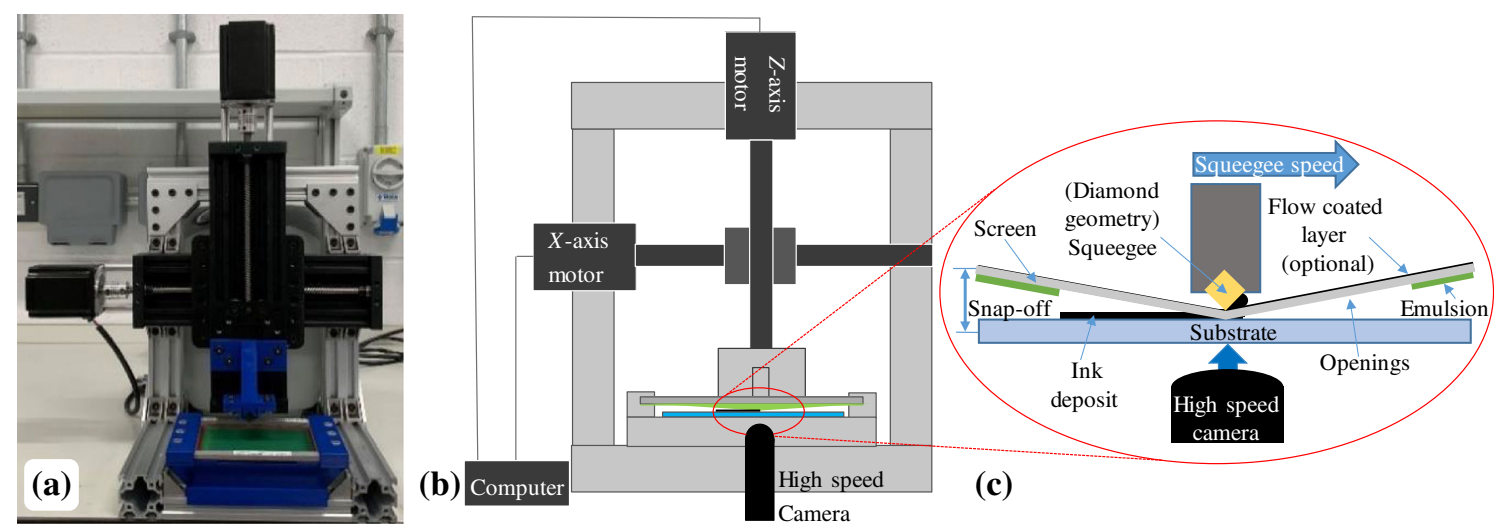

Fig. 1: Photograph (a) and schematic diagram (b) of the screen-printing simulation rig, showing the positioning of the highspeed camera. The zoomed in schematic diagram (c) shows a cross section through the point of contact during screen printing, as would be observed by the high-speed camera

which an average and standard deviation could be calculated. The filamentation and separation modes occurring were also assessed.

\section{Printed line characterization}

White light interferometry (NT9300, Veeco Instruments, Inc., Plainview, NY, USA) was used to measure a full three-dimensional surface profile of the printed lines. The ink film thickness was calculated as the difference between the average height of the printed line and the average height of the surrounding substrate. Five-times magnification was used, giving a measurement area of $1.2 \mathrm{~mm}$ by $0.93 \mathrm{~mm}$ (at a resolution of $736 \times 480$ pixels with sampling at 1.67$\mu \mathrm{m}$ intervals). Lines were each measured in four evenly spaced points, with three print samples assessed for each parameter setting, totaling 12 measurements from which the average and standard deviation was calculated. Geometric features were assessed by taking discrete measurements over the 1.2-mm-length sections measured by the interferometer (736 measurements at $1.67-\mu \mathrm{m}$ intervals for each measured section). Standard deviations in line width and thickness were calculated.

To provide an overall image of the printed lines produced on the rig, optical microscopy (Alicona Infinite Focus G5 microscope, Alicona Imaging $\mathrm{GmbH}$ ) was used for its ability to more effectively capture the surface form of carbon ink in true color.

Two-point probe measurements were conducted with a Keithley 2400 digital Sourcemeter on the 6$\mathrm{mm}$-long 200- $\mu \mathrm{m}$ lines, to provide a comparison of the line resistance of the lines produced. Three measurements were conducted for each of the parameter settings assessed, from which an average and standard deviation was calculated.

\section{Results}

\section{Rheological and extensional testing}

Figure 2 shows the viscosity profile during increasing and then decreasing shear (a) and viscoelastic profiles (b). The ink was highly shear thinning, with a reduction in viscosity as the shear stress was increased, particularly from 1 to $50 \mathrm{~s}^{-1}$. It also showed relatively little hysteresis between 10 and $100 \mathrm{~s}^{-1}$, where the same viscosities were produced both when shear rates were increased up to $100 \mathrm{~s}^{-1}$ and when they were reduced back from $100 \mathrm{~s}^{-1}$. This would be due to the ink recovering quickly enough that the viscosities produced at these shear rates would not be affected by higher shear rates tested previously.

Both the elastic component (storage modulus, $G^{\prime}$ ) and viscous component (loss modulus, $G^{\prime \prime}$ ) of the shear modulus increased with frequency. $G^{\prime}$ was consistently larger than $G^{\prime \prime}$ for the frequencies tested. Although the gap between the two decreased at higher frequencies, this produced a relatively low phase angle $(\delta)$, which is around $27^{\circ}$ at a frequency of $1 \mathrm{~Hz}$. This suggested that the ink behaved in an elastic manner for the frequencies tested. At higher frequencies, it was possible that the $G^{\prime \prime}$ would become greater than the $G^{\prime}$. However, this would be above the frequency range relevant to the screen-printing process being used.

The separation mechanism and the relative partition of ink between top and bottom plates during the CaBER tests are shown as a function of separation 

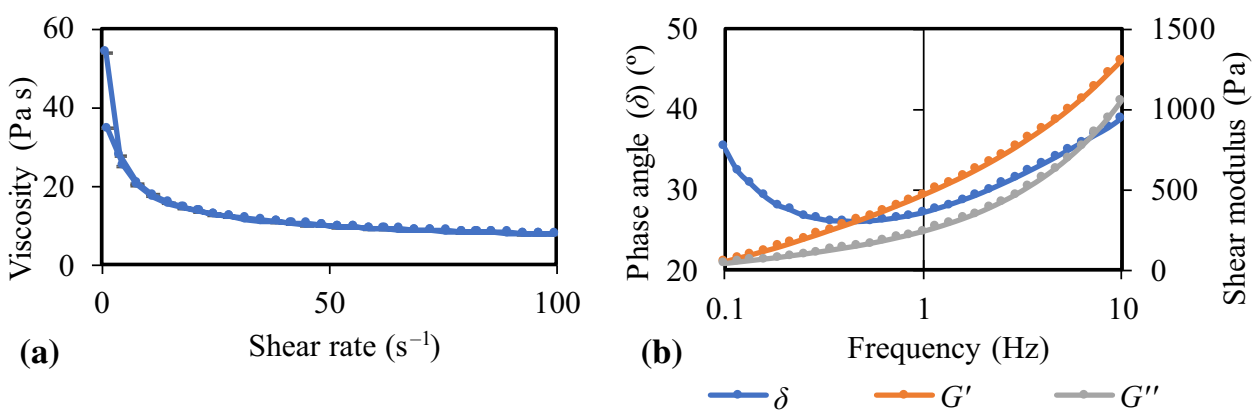

Fig. 2: Viscosity profile (a) and viscoelastic profile assessing changes in phase angle $(\delta)$, storage modulus ( $\left.G^{\prime}\right)$ and loss modulus $\left(G^{\prime \prime}\right)$ with frequency $(b)$ for the carbon ink assessed

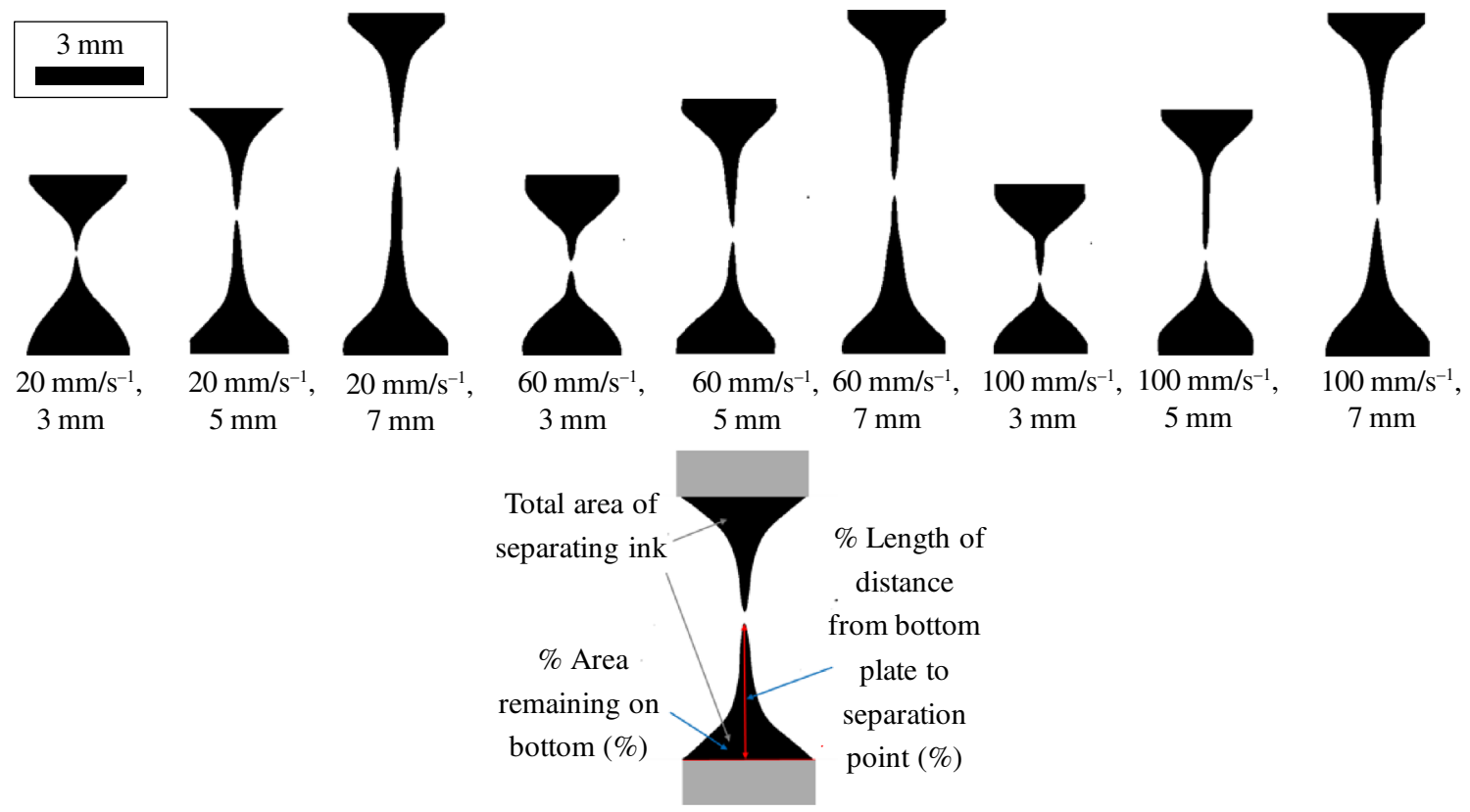

Fig. 3: High-speed images of separation at the point of film splitting with separation speed and separation distance

distance and separation speed (Fig. 3). The percentage partition of ink between top and bottom plates is described in terms of the cross-sectional area at the point of separation of the ink on the lower plate compared with the total area and length to separation from the bottom plate (Fig. 4). Between $45 \%$ and $60 \%$ of the ink was apportioned to the bottom plate. As separation speed was increased, the relative amount of ink remaining on the bottom plate decreased for all separation distances tested. However, the extent of change varied with the separation distances. The 7-mm gap had the largest reduction of $11 \%$, and the $5-\mathrm{mm}$ gap had the smallest reduction of $4 \%$ of the total area of ink being separated. Although there was an overall increase in percentage of ink remaining on the bottom when increasing separation distance from 3 to $7 \mathrm{~mm}$, there was a reduction when increasing from 3 to $5 \mathrm{~mm}$ for all speeds tested. For all speeds tested (Fig. 4), the length from the bottom plate to the separation point was found to decrease linearly with speed. This was due to the elastic nature of the ink causing it to pull up more toward the top plate with the increasing strain rate.

When comparing these trends at the moment of separation (Fig. 3), the position at which separation occurs reduced with increasing separation speed. The separation position was near the center of the filamentation region at separation speeds of $20 \mathrm{~mm}$ $\mathrm{s}^{-1}$, moving to below the center point at $100 \mathrm{~mm} \mathrm{~s}^{-1}$. The capillary thinning breakup mode of the ink was similar to a power law fluid, where the ink filaments formed hourglass-like profiles prior to separation. However, higher speeds and gaps exhibited profiles more similar to a weakly elastic fluid, exhibiting longer necking regions and more homogeneous extensional flow occurring over the filament. This was due to the strong shear thinning and viscoelastic nature of the ink, where increases in the strain and strain rate caused the ink to exhibit more elastic behaviors. 

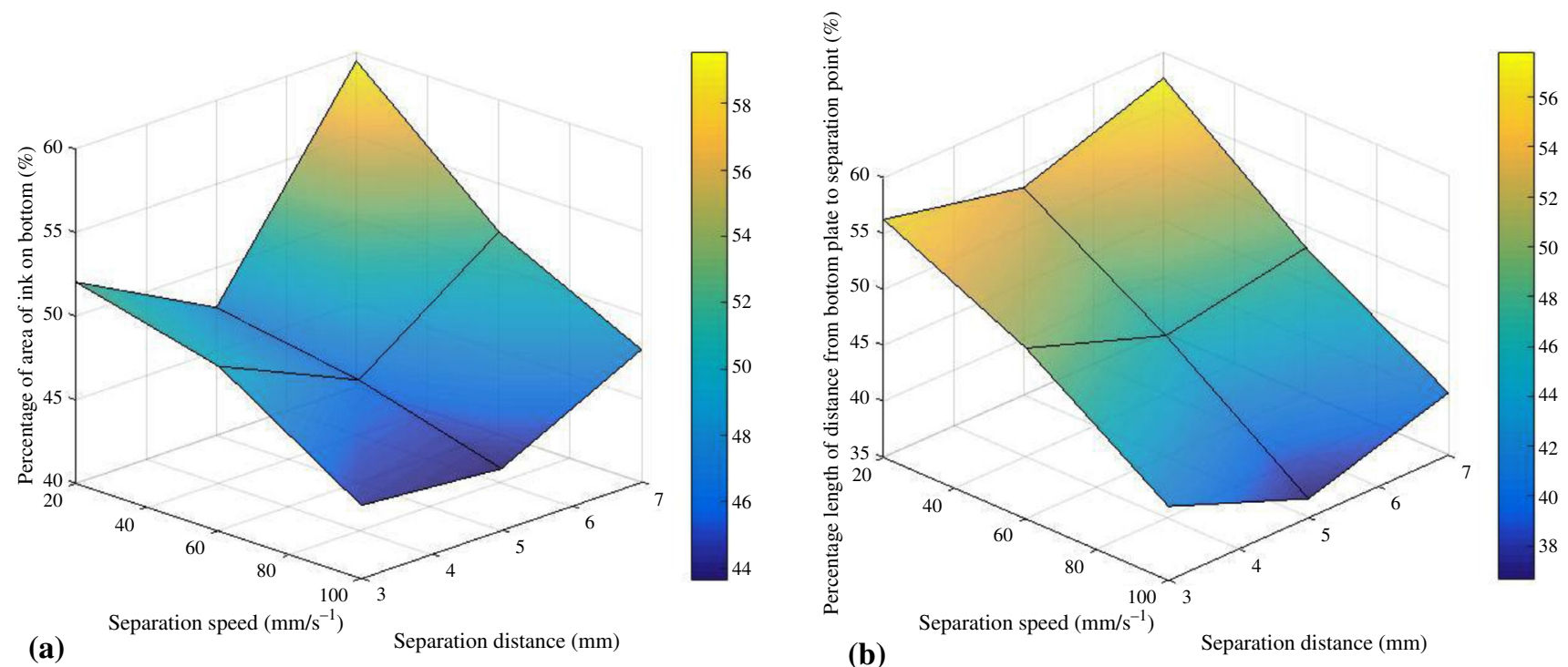

Fig. 4: Surface response of the percentage of the cross-sectional area of ink remaining on the bottom plate (a) and percentage length to separation point from the bottom plate (b) with changes in separation distance and separation speed

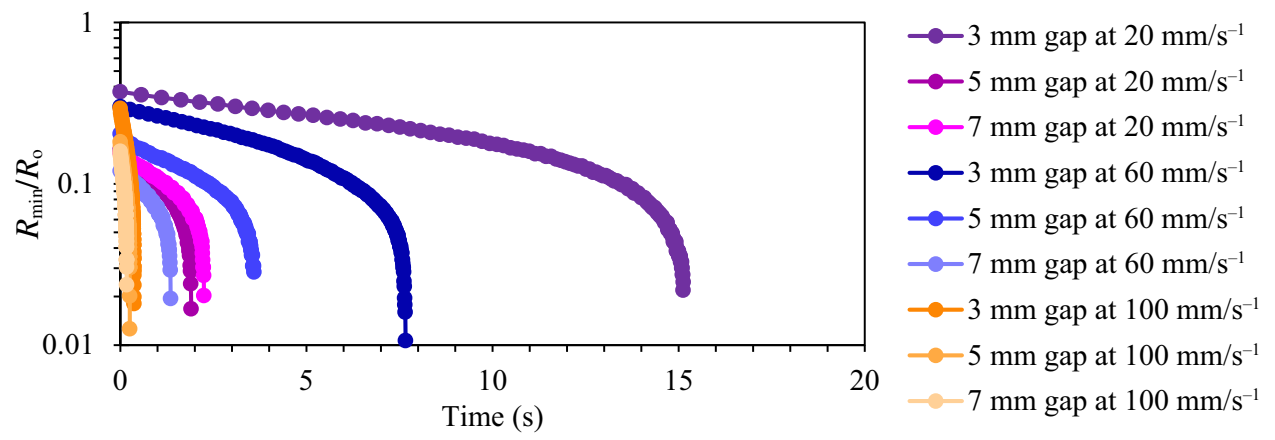

Fig. 5: Evolution of the minimum radius

The change in the minimum diameter over time for each of the tests [expressed as the ratio of the final diameter divided by the initial diameter $\left.\left(R_{\min } / R_{\mathrm{O}}\right)\right]$ is shown in Fig. 5. The smallest diameter in each case represents the point at which the ink film splits. The slowest speed over the shortest distance took the longest time to split, whereas the ink separated over the greatest distance at the highest rate took the least time to split. All inks separated at the highest rate of $100 \mathrm{~mm} \mathrm{~s}^{-1}$ took less time to split than those conducted at slower separation rates, for all separation distances. The time taken to separate decreased with the increase in separation distance. However, there were some overlaps in the results conducted at 20 and $60 \mathrm{~mm} \mathrm{~s}^{-1}$, where the inks separated at $20 \mathrm{~mm} \mathrm{~s}^{-1}$ at separation distances of 7 and $5 \mathrm{~mm}$ took less time to split than those separated at $60 \mathrm{~mm} \mathrm{~s}^{-1}$ over distances of 5 and $3 \mathrm{~mm}$.

The changes in strain (distance) and strain rate (separation speed) had clear effects on the ink separation mechanism and duration. Increases in separation speed led to a greater preference for ink to separate toward the top rather than bottom plate. The highest separation gap produced a similar effect.

\section{Assessment of print contact regions and print characterization}

\section{Classification of flow regions during screen printing}

The images from the high-speed camera on the screenprinting rig allow the ink deposition process to be split up into quantifiable regions. The regions are based on the four key stages of the transfer of the ink to the substrate identified by Messerschmitt, ${ }^{26}$ along with the length of the paste flow region ahead of the squeegee (Fig. 6). The print direction is from left to right, and a mirror image can be seen beneath the point of contact from the reflection on the surface of the substrate. After the squeegee has brought the mesh into contact with the substrate, the ink can then pass through the gaps in the mesh. During the adhesion stage, the ink forms a continuous bridge between the mesh and 

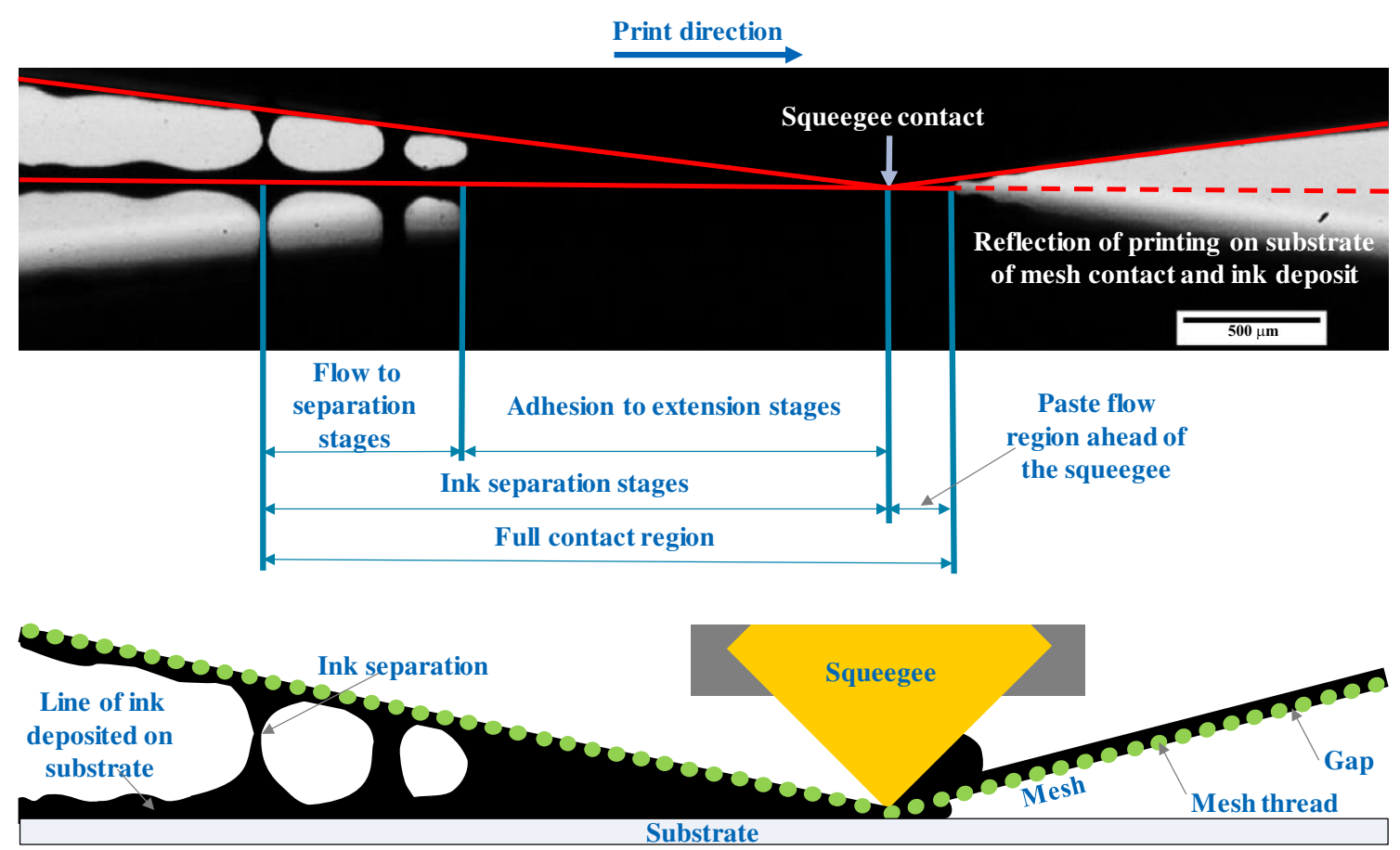

Fig. 6: Labeled high-speed camera image of the squeegee forcing the mesh into contact with the substrate, where the ink is deposited from the mesh to the substrate as the squeegee flows across the screen (above) along with a cross-sectional schematic diagram of the process (below)

substrate directly behind the squeegee contact point. This continues in the extension stage where the ink remains in continuous contact, but is stretched over an increasing distance while the mesh moves away from the substrate. The flow stage is where the ink starts to separate from the main body of ink behind the squeegee and forms filaments. These then start to display a localized rate of thinning, known as "necking," ${ }^{11}$ which then leads to complete separation. These four stages together make up the ink separation stages. The paste flow region ahead of the squeegee was also measured for completeness. In practice, the transitions between stages 1 and 2 and stages 3 and 4 are difficult to measure, so characterization is in terms of adhesionto-extension stages (stages 1 and 2) and flow-toseparation stages (stages 3 and 4). The overall length of these combined stages where the ink is in simultaneous contact with the mesh and substrate is labeled as the full contact region.

The snap-off distance had a greater effect on the lengths of the different flow regions than the squeegee speed (Fig. 7). Reducing the snap-off distances led to significant increases in the length of the adhesion-toextension stages, where ink behind the squeegee contact point was in simultaneous contact with both the mesh and substrate without any gaps in the body of ink. This stage increased from an average of $1617 \mu \mathrm{m}$ at a snap-off distance of $1.825 \mathrm{~mm}$ to $3328 \mu \mathrm{m}$ when snap-off distance reduced to $1.125 \mathrm{~mm}$. As the distance between mesh and substrate was lowered from 1.825 to $1.125 \mathrm{~mm}$, the angle between the mesh and substrate at contact point was reduced from $6^{\circ}$ to $4^{\circ}$. As a result, the critical vertical distance required for the ink to reach sufficient shear for splitting and forming filaments was not reached until a greater distance behind the squeegee contact point. The flow-to-separation stages, where filaments formed and then separated, were much shorter in length than the adhesion-toextension stage lengths. Although there was an increase in the length of these stages, from an average of 201 to $698 \mu \mathrm{m}$, snap-off was reduced from 1.825 to $1.125 \mathrm{~mm}$, respectively.

When print speed was increased, there was an increase in the length of the adhesion-to-extension stages, increasing from $1410 \mu \mathrm{m}$ to $2304 \mu \mathrm{m}$ as speed was increased from 100 to $500 \mathrm{~mm} \mathrm{~min}^{-1}(1.7-8.3 \mathrm{~mm}$ $\mathrm{s}^{-1}$ ). There was a reduction in the length of the flow-toseparation stages from 465 to $177 \mu \mathrm{m}$ as speeds increased from 100 to $500 \mathrm{~mm} \mathrm{~min}{ }^{-1}$. However, there was an overall increase in the length of the full contact region with increases in speed, from an average of $2218 \mu \mathrm{m}$ at $100 \mathrm{~mm} \mathrm{~min}^{-1}$ to $2783 \mu \mathrm{m}$ at $500 \mathrm{~mm}$ $\min ^{-1}$. Although, there was a slight reduction in the full contact region length to $2158 \mu \mathrm{m}$ when increasing speed from $100 \mathrm{~mm} \mathrm{~min}^{-1}$ to $300 \mathrm{~mm} \mathrm{m^{-1 }}(1.7 \mathrm{~mm}$ $\mathrm{s}^{-1}$ to $5.0 \mathrm{~mm} \mathrm{~s}^{-1}$ ).

\section{Assessment of filamentation behavior}

Consecutive images are compared at different snap-off distances and speeds (Fig. 8). There was little effect of 

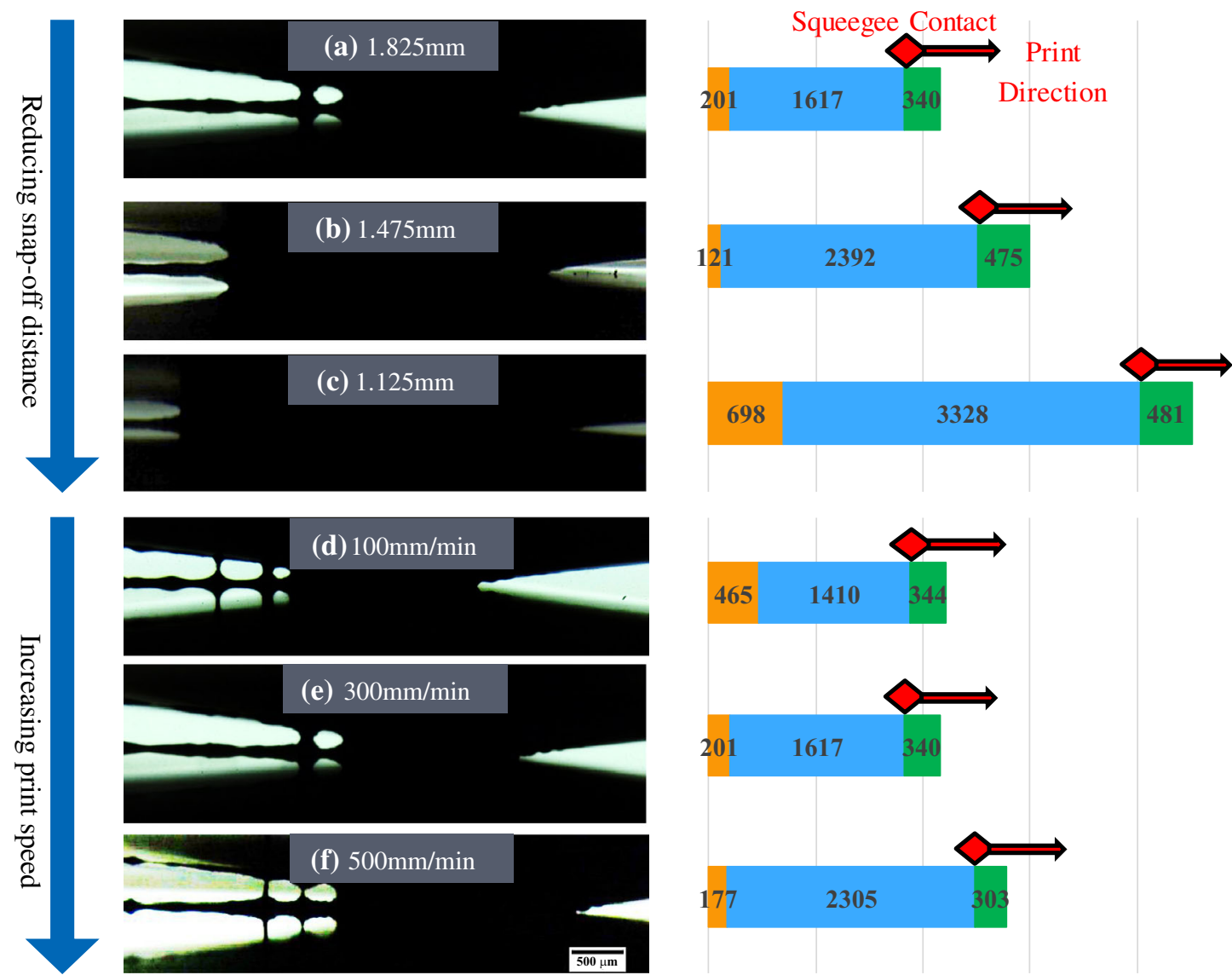

0

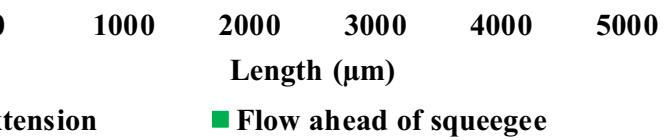

Fig. 7: Quantification of print stage lengths for deposition using varying snap-off distances at fixed print speed of $300 \mathrm{~mm} \mathrm{~min}-1(a-c)$ and at a range of print speeds using a fixed gap of $1.825 \mathrm{~mm}(\mathrm{~d}-\mathrm{f})$

snap-off distance on the number of filaments which formed and separated behind the continuous body of elongating ink. The number of separation points across the $5 \mathrm{~mm}$ length of print assessed ranged from 2 to 5 . The length between these separating points ranged between 965 and $3618 \mu \mathrm{m}$. Over the print duration for the 1.475 and $1.125 \mathrm{~mm}$ snap-off distances, there was the main body of ink in the extension flow region and one filamentation region. The average length of the average flow-to-separation region was far smaller than the average distance between separation points.

For the prints conducted at the higher squeegee speeds, the filaments formed a long necking region along the center of the filament, and this had capillary thinning and breakup which occurs with elastic fluids. ${ }^{32}$ This was also seen in the extensional testing at higher speeds. At slower speeds, both the filaments formed during printing and on the extensional rheology tests displayed a mode of capillary thinning and breakup similar to the profile of a power law fluid. When increasing the print speed, strain rate of the ink caused it to behave as an increasingly elastic fluid. The increase in print speed also corresponded with a reduction in the number and frequency of filaments and separation points visible as the mesh and substrate separated. For example, as speed was increased from 100 to 300 then $500 \mathrm{~mm} \mathrm{~min}^{-1}$, the number of filaments visible in the image fell from 10 to 5 to 4 .

\section{Topography of prints}

The optical images (Figs. 9a, 9b and 9c) showed little variation in the evenness and waviness of the edges of the printed lines with snap-off distance. When comparing these results with the length of the flow regions occurring during the print, the snap-off distance of $1.475 \mathrm{~mm}$ produced fewer filaments and a shorter flowto-separation region than the $1.825 \mathrm{~mm}$ snap-off distance (Fig. 7). It also produced narrower filaments than the $1.125-\mathrm{mm}$ separation distance, where the larger filaments led to more significant bumps on the surface 
(a) $1.475 \mathrm{~mm}$ snap, $300 \mathrm{~mm} / \mathrm{min}$ speed

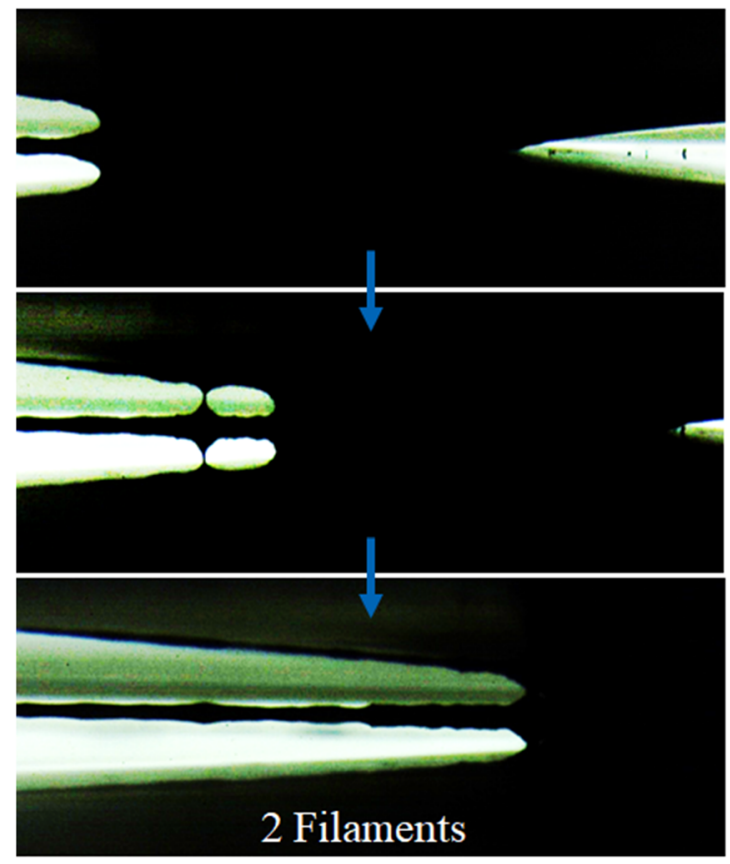

(c) $1.825 \mathrm{~mm}$ snap, $100 \mathrm{~mm} / \mathrm{min}$ speed

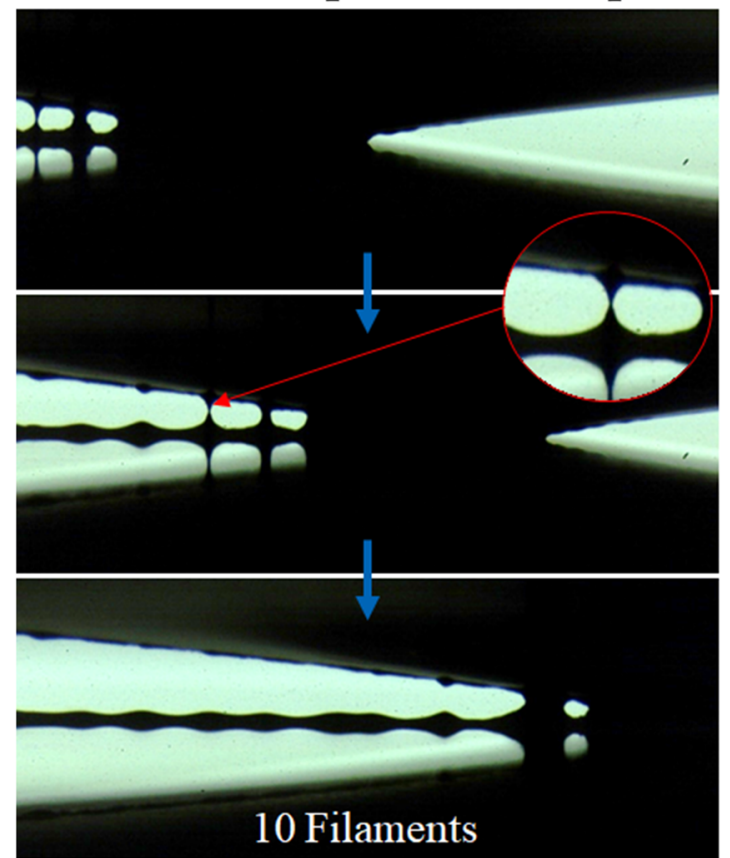

(b) $1.125 \mathrm{~mm}$ snap, $300 \mathrm{~mm} / \mathrm{min}$

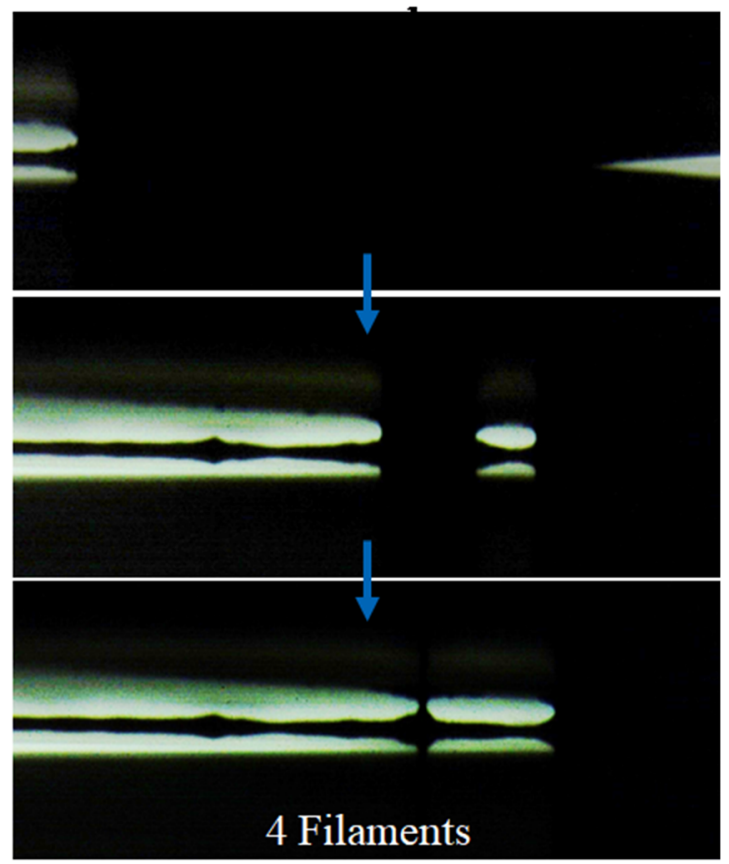

(d) $1.825 \mathrm{~mm}$ snap, $500 \mathrm{~mm} / \mathrm{min}$ speed

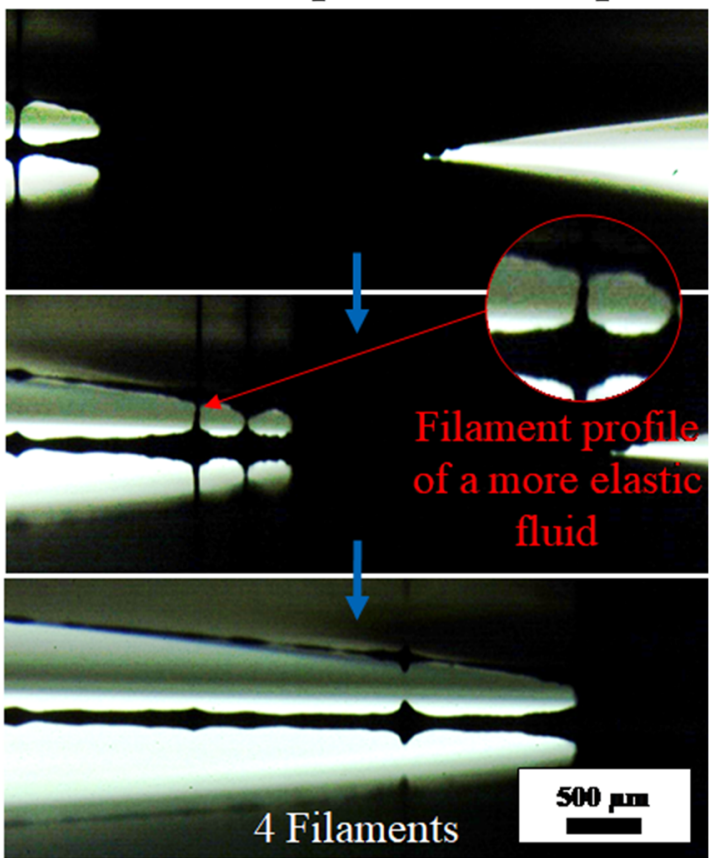

Fig. 8: High-speed camera images at $5 \times$ magnification of different stages of the deposition of the $200-\mu \mathrm{m}$ line conducted at $300 \mathrm{~mm} \mathrm{~min} \mathrm{mi}^{-1}$ with snap-off distances of (a) $1.475 \mathrm{~mm}$ and (b) $1.125 \mathrm{~mm}$ as well as conducted with a snap-off distance of $1.825 \mathrm{~mm}$ with squeegee speeds of (c) $100 \mathrm{~mm} \mathrm{~min}^{-1}$ and (d) $500 \mathrm{~mm} \mathrm{~min}^{-1}$

of the line conducted at the lowest snap-off distance (Fig. 8).

The average film thickness and width of the lines produced varied both with changes in snap-off distance and squeegee speed (Fig. 10). The mid-range snap-off distance of $1.475 \mathrm{~mm}$ produced the greatest film thickness and line width at 13.6 and $316 \mu \mathrm{m}$, respectively. The lowest film thickness and line width, of 12.4 and $307 \mu \mathrm{m}$, respectively, were produced at the highest snap-off distance. In terms of print speed, the lowest 


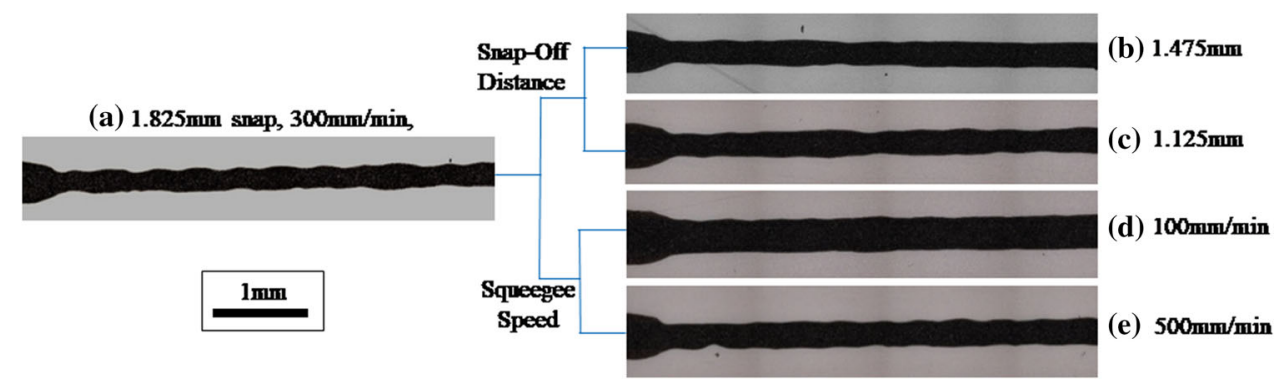

Fig. 9: Changes in the optical microscopy images (10x magnification) of the printed lines with standard settings (300 $\mathrm{mm} \mathrm{min}^{-1}, 1.825 \mathrm{~mm}$ snap-off distance and at contact point) (a), with the snap-off distance reduced to $1.475 \mu \mathrm{m}$ (b) and $1.125 \mu \mathrm{m}$ (c) as well as with the print speed reduced to $100 \mathrm{~mm} \mathrm{~min}^{-1}$ (d) and increased to $500 \mathrm{~mm} \mathrm{~min}^{-1}$ (e)
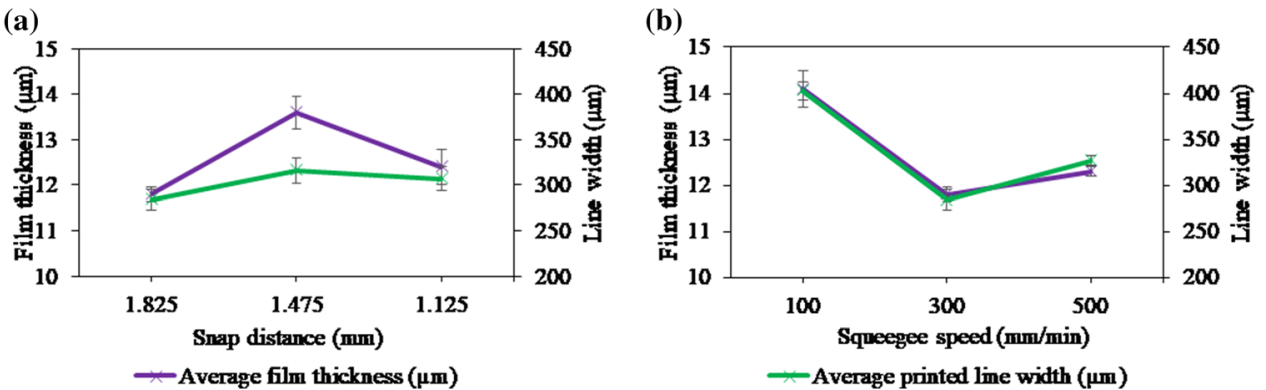

Fig. 10: Variations in the average film thickness and average printed line width with changes in snap-off distance (a) and squeegee speed (b) (analyzed using white light interferometry) (error bars represent standard deviation)
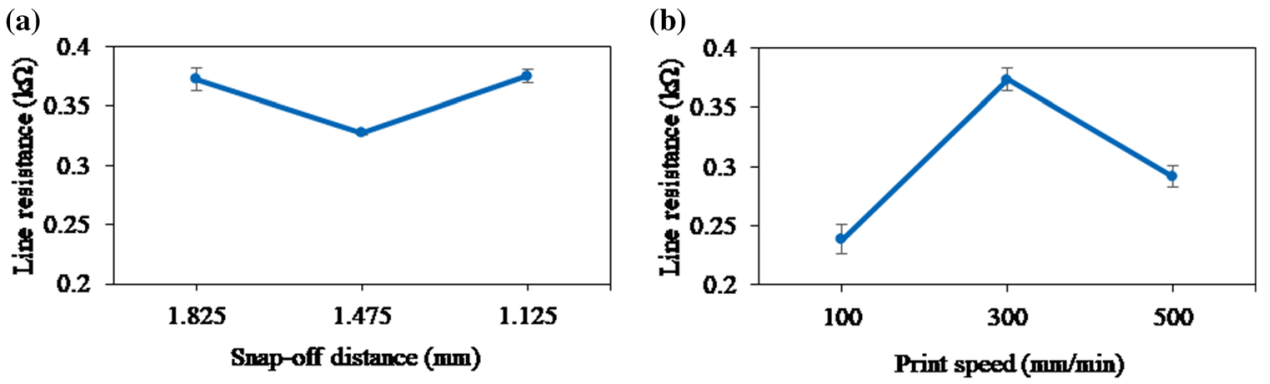

Fig. 11: Changes in line resistance of the 200- $\mu \mathrm{m}$ lines with changes in snap-off distance (a) and speed (b) (error bars represent standard deviation)

speed of $100 \mathrm{~mm} \mathrm{~min}^{-1}$ gave the highest ink film thickness and line width of 14.1 and $403 \mu \mathrm{m}$, respectively, out of the speeds assessed. It also produced the most consistent and least wavy line edge. The print produced at $300 \mathrm{~mm} \mathrm{~min}^{-1}$ gave the lowest film thickness and line width (12.4 and $307 \mu \mathrm{m}$, respectively). A further increase in print speed to $500 \mathrm{~mm}$ $\min ^{-1}$ gave a slight increase in deposition. The reduction in deposition with increased print speed, and therefore separation speed, corresponded with the CaBER test, where increases in separation speed led to less ink remaining on the bottom plate. As well as in this, the filamentation breakup started forming longer, more elastic necking regions.

\section{Electrical comparison of prints}

The changes in line resistance of the $200-\mu \mathrm{m}$ printed lines are shown in Fig. 11. The results are inversely related to the topography profiles of the prints (Fig. 9), where the greater ink deposits, with larger line widths and film thicknesses, had lower line resistance and therefore higher conductivity. The snap-off distance of 
$1.825 \mathrm{~mm}$ with squeegee speed of $100 \mathrm{~mm} \mathrm{~min}^{-1}$, which produced the greatest ink deposit, produced the lowest line resistance of $0.239 \mathrm{k} \Omega$.

\section{Discussion}

There is agreement with the qualitative theory of four stages of ink deposition described by Messerschmitt, where the separation forces induce a flow causing a shearing action which leads to the ink splitting. ${ }^{26}$ This study quantified the relative length and duration of each of the four stages. The flow-to-separation stages were significantly shorter than the adhesion-to-extension stages for all parameter settings, ranging from 122 to $698 \mu \mathrm{m}$ in length. The adhesion-to-extension lengths ranged from 1410 to $3328 \mu \mathrm{m}$ and were most affected by the snap-off distance. The reducing snap-off distance led to reductions in the angle between the mesh and substrate, which prolonged the adhesion-to-extension stages. The body of ink behind the squeegee took longer to reach the length required for sufficient shear flow to induce ink splitting. For increases in print speed, there was a reduction in the lengths of the flowto-separation stages, with a gradual increase in the adhesion-to-extension stage lengths. This also led to notable changes in the mode of capillary thinning and breakup occurring during ink filamentation and subsequent separation. The ink exhibited flow profiles characteristic of elastic fluids at higher speeds, while showing necking profiles more characteristic of power law fluids at lower speeds. This corresponded with the extensional rheology profiles which also found that the filaments necking profiles went from being characteristic of a power law fluid to an elastic fluid at comparable separation speeds to the print speeds used.

Previous studies found increasing snap-off distance led to greater ink deposits for a range of ink viscosities. ${ }^{14}$ However, other studies found there to be an optimal snap-off distance for a given ink and substrate, ${ }^{15}$ while others again found the snap-off distance had no significant effects on the print quality of fine lines. ${ }^{12}$ In the case of squeegee speed, increases in print speed led to improvements in print quality. ${ }^{13,14,16,17}$ However, others found this was only the case up to an optimal value for a given ink and substrate. ${ }^{15}$ Some reported increases in print speed led to reductions in print quality. ${ }^{12,18}$ Overall, this suggests the effect of snap-off distance and squeegee speed depends on their interaction with other parameters, such as ink rheology. There may also be nonlinear trends with changes in print topography and performance with snap-off distance and speed.

In this study, the snap-off distance was found to have nonlinear effects on print topography and line resistance, with the medium snap-off distance producing the greatest ink deposit and lowest line resistance. There was an overall reduction in the average ink deposited with increases in print speed, with corre- sponding increases in line resistance. Higher separation speeds were also found to cause less ink to remain on the bottom plate in the CaBER tests, with changes in the capillary thinning and breakup mode. The ink was found to behave more elastically with increases in strain rate. Assessing changes in the vertical strain rate with extensional rheology tests can indicate how the flow-to-separation flow regions of the screen-printing process can be influenced by separation speed. However, imaging of the full deposition process is also required as printing consists of simultaneous shearing and extensional forces, as well as time-dependent phenomena.

High print speeds can reduce the viscosity of the ink and display the inherent elasticity of the ink, as demonstrated in the extensional rheology tests. A significant time was required for recovery, which was substantially higher than the time frames involved in the print cycle. This might negate some of the speed and separation effects seen in purely extensional tests, which do not pre-thin the ink in this way, and thus give fewer clear trends in filamentation behaviors. Overall, the findings in the literature along with those presented in this research suggest that the extent to which the print settings play a role may be influenced by the viscoelasticity and pseudoplasticity of the ink.

Carbon ink is typically used for relatively large area features, from carbon electrodes for sensors to panels for photovoltaic applications. The consistency of the printed pattern or feature is most important for electrical performance, with the edge resolution being less important. The relatively slow recovery of the ink after shearing resulted in slumping of the ink after deposition. Therefore, the printed lines were wider than the nominal line width of the screen section through which they were printed. Further work could be conducted to see whether similar flow mechanisms are present for inks with different rheological properties.

\section{Conclusion}

The impact of snap-off distance and squeegee speed on the deposition mechanism of a carbon conductive ink by screen printing has been investigated using a range of techniques including high-speed imaging and print analysis. Advanced rheology techniques have also been used to evaluate the ink.

The theory proposed qualitatively by Messerschmitt of four identifiable stages of ink deposition was quantified for this case of a conductive carbon ink. Altering the squeegee print speed and snap-off distance led to variations in the lengths of the ink deposition stages. Reductions in the snap-off distance led to significant increases in the adhesion-to-extension stages. The smaller snap-off distances caused reductions in the angle between the mesh and substrate. Sufficient shear flow for separating the ink was not achieved until there was a greater snap-off distance 
behind the squeegee. Changes in print speed led to alterations in the necking profiles formed during the filamentation stage. The ink acted in a more elastic manner at higher speeds. This complimented the extensional rheology results which observed similar changes in the filamentation profile and also found less ink remaining on the lower plate as separation speed was increased. The changes in snap-off distance and print speed also led to changes in the print topography produced.

As well as improve the understanding of how to optimize print quality and performance of printed electronics, these results could be used to develop predictive methods and provide boundary conditions for mathematical models.

Acknowledgments The authors would like to acknowledge the funding from the European Social Fund via the Welsh Government, the Engineering and Physical Sciences Research Council (Grant Reference: EP/L015099/1) through the Materials and Manufacturing Academy (M2A) Doctoral Training Scheme and icmPrint that has made this research possible.

Conflict of interest There are no conflicts of interest.

Open Access This article is distributed under the terms of the Creative Commons Attribution 4.0 International License (http://creativecommons.org/lice nses/by/4.0/), which permits unrestricted use, distribution, and reproduction in any medium, provided you give appropriate credit to the original author(s) and the source, provide a link to the Creative Commons license, and indicate if changes were made.

\section{References}

1. Philip, B, Jewell, E, Greenwood, P, Weirman, C, “Material and Process Optimization Screen Printing Carbon Graphite Pastes for Mass Production of Heating Elements." J. Manuf. Process., 22 (1) 185-191 (2016). https://doi.org/10.1016/j.jma pro.2016.03.001

2. Tehrani, Z, Burwell, G, Azmi, MAM, Castaing, A, Rickman, R, Almarashi, J, et al., "Generic Epitaxial Graphene Biosensors for Ultrasensitive Detection of Cancer Risk Biomarker." 2D Mater, 1 (2) 025004 (2014)

3. Tehrani, Z, Korochkina, T, Govindarajan, S, Thomas, DJ, Mahony, JO, Kettle, J, et al., "Ultra-Thin Flexible Screen Printed Rechargeable Polymer Battery for Wearable Electronic Applications." Org. Electron., 26 (1) 386-394 (2015). https://doi.org/10.1016/j.orgel.2015.08.007

4. Baker, J, Hooper, K, Meroni, S, Pockett, A, McGettrick, J, Wei, Z, et al., "High Throughput Fabrication of Mesoporous Carbon Perovskite Solar Cells." J. Mater. Chem. A., 5 (35) 18643-18650 (2017)
5. Zhang, L, Liu, T, Liu, L, Hu, M, Yang, Y, Mei, A, et al., "The Effect of Carbon Counter Electrodes on Fully Printable Mesoscopic Perovskite Solar Cells." J. Mater. Chem. A., 3 (17) 9165-9170 (2015)

6. Zabek, D, Seunarine, K, Spacie, C, Bowen, C, "Graphene Ink Laminate Structures on Poly(vinylidene difluoride) (PVDF) for Pyroelectric Thermal Energy Harvesting and Waste Heat Recovery." ACS Appl. Mater. Interfaces, 9 (10) 9161-9167 (2017)

7. Cao, Z, Koukharenko, E, Tudor, MJJ, Torah, RNN, Beeby, SPP, "Flexible Screen Printed Thermoelectric Generator with Enhanced Processes and Materials." Sens. Actuators A Phys., 238 196-206 (2016). https://doi.org/10.1016/j.sna.2015. 12.016

8. Jewell, E, Philip, B, Greenwood, P, “Improved Manufacturing Performance of Screen Printed Carbon Electrodes through Material Formulation." Biosensors, 6 (3) 30 (2016)

9. Philip, B, Jewell, E, Worsley, D, "The Impact of Solvent Characteristics on Performance and Process Stability of Printed Carbon Resistive Materials." J. Coat. Technol. Res., 13 (5) 911-920 (2016)

10. Jewell, E, Hamblyn, S, Claypole, T, Gethin, D, "Deposition of High Conductivity Low Silver Content Materials by Screen Printing." Coatings, 5 (2) 172-185 (2015)

11. Jewell, EH, Hamblyn, SM, Claypole, TC, Gethin, DT, "The Impact of Carbon Content and Mesh on the Characteristics of Screen Printed Conductive Structures." Circuit World, 39 (1) 13-21 (2013)

12. Pan, J, Tonkay, GL, Quintero, A, Ave, WP, "Screen Printing Process Design of Experiments for Fine Line Printing of Thick Film Ceramic Substrates." J. Electron. Manuf., 9 (3) 203-213 (1999)

13. Molamphy, TA, Stephenson, MI, Murphy, EA, “Application of Experimental Design to the Solder Paste Screen Printing Process." Solder Surf. Mt. Technol., 11 4-6 (1992)

14. Jewell, EH, Claypole, TC, Gethin, DT, “The Impact of Ink Rheology on the Image Transfer Mechanism in Screen Printing." In: Proceedings of Institute of Non Newtonian Fluid Mechanics. Cardiff (2003)

15. Cao, K, Cheng, K, Wang, Z. Optimization of Screen Printing Process. In: IEEE, 7th International Conference on Electronics Packaging Technology. (2006) [cited 2016 Jan 27]. Accessed at: https://www.google.co.uk/search?q=Optimizati on + of + Screen + Printing + Process\&oq $=$ Optimization + of + Scr een + Printing + Process \&aqs $=$ chrome..69i57.525j0j4\&sourcei $\mathrm{d}=$ chrome\&es_sm $=93 \& \mathrm{ie}=\mathrm{UTF}-8$

16. Fox, IJ, Bohan, MFJ, Claypole, TC, Gethin, DT, "Film Thickness Prediction in Halftone Screen-Printing." Proc. Inst. Mech. Eng. Part E J. Process. Mech. Eng., 217 345-359 (2003). https://doi.org/10.1243/095440803322611705

17. Mannan, SH, Ekere, NN, Ismail, I, Lo, EK, "Squeegee Deformation Study In The Stencil Printing Of Solder Pastes." IEEE Trans. Compon. Packag. Manuf. Technol. Part A., 17 (3) 470-476 (1994)

18. Jewell, EH, Claypole, TC, Gethin, DT, "Viscosity Control in the Screen Printing of Ceramic Transfers." Surf. Coat. Int. Part B Coat. Trans., 86 (2) 155-163 (2003). https://doi.org/10. 1007/BF02699628

19. Riemer, DE, "The Direct Emulsion Screen as a Tool for High Resolution Thick Film Printing." In: Electronic Component Conference Proceedings, Washington, DC, USA, pp. 463-467 (1971) 
20. Riemer, DE, "The Theoretical Fundamentals of the Screen Printing Process." Microelectron. Int., 6 (1) 8-17 (1989)

21. Riemer, DE. Ein Beitrag zur Untersuchung der PhysikalischTechnischen Grundlagen des Siebdruckverfahrens. Technische Universität Berlin (1988)

22. Fox, IJ, Claypole, TC, Gethin, DT, "An Experimental Investigation into Ink Transfer Using a Roller Squeegee in High-Speed Screen Printing." Proc. Inst. Mech. Eng. E, J. Process. Mech. Eng., 217 (E4) 307-321 (2003)

23. $\mathrm{Xu}, \mathrm{C}$, Willenbacher, N, "How Rheological Properties Affect Fine-Line Screen Printing of Pastes: A Combined Rheological and High-Speed Video Imaging Study." J. Coat. Technol. Res., 15 (6) 1401-1412 (2018). https://doi.org/10.10 07/s11998-018-0091-2

24. Riemer, DE, "Analytical Engineering Model of the Screen Printing Process: Part I.” Solid State Technol., 8 (1) 107-111 (1988)

25. Riemer, DE, "Analytical Engineering Model of the Screen Printing Process: Part II." Solid State Technol., 9 (1) 85-90 (1988)

26. Messerschmitt, E, "Rheological Considerations for Screen Printing Inks.” Screen Print., 72 (10) 62-65 (1982)

27. Kapur, N, Abbott, SJ, Dolden, ED, Gaskel, PH, "Predicting the Behavior of Screen Printing." IEEE Trans. Compon. Packag. Manuf. Technol., 3 (3) 508-515 (2013). https://ww w.google.co.uk/_chrome/newtab?espv=2\&ie=UTF-8
28. Dimic-Misic, K, Hummel, M, Paltakari, J, Sixta, H, Maloney, T, Gane, P, "From Colloidal Spheres to Nanofibrils: Extensional Flow Properties of Mineral Pigment and Mixtures with Micro and Nanofibrils Under Progressive Double Layer Suppression." J. Colloid Interface Sci., 446 31-43 (2015). https://doi.org/10.1016/j.jcis.2015.01.004

29. Anna, SL, Mckinley, GH, "Elasto-Capillary Thinning and Breakup of Model Elastic Liquids." J. Rheol., 45 (1) 115-138 (2001)

30. Rueden, CT, Schindelin, J, Hiner, MC, Dezonia, BE, Walter, AE, Arena, ET, et al., "ImageJ2: ImageJ for the Next Generation of Scientific Image Data." BMC Bioinf., 18 1-26 (2017)

31. Mckinley, GH, Anna, SL, Tripathi, A, Yao, M, "Extensional Rheometry of Polymeric Fluids and the Uniaxial Elongation of Viscoelastic Filaments." (1999). Accessed 22 May 2019 at: http://web.mit.edu/nnf/publications/GHMconf1.pdf

32. McKinley, GH, "Visco-Elasto-Capillary Thinning and Break-Up of Complex Fluids." Rheol. Rev. (2005). http://m it.dspace.org/handle/1721.1/18085

Publisher's Note Springer Nature remains neutral with regard to jurisdictional claims in published maps and institutional affiliations. 